\title{
Hérnia discal associada a cisto discogênico: Considerações sobre o diagnóstico e a patogênese
}

\author{
Sérgio Listik*, Marcelo Ferraz de Campos*, Gilles Landman**, \\ Antônio José da Rocha*** \\ Serviço de Neurocirurgia do Hospital São Cristovão. São Paulo, SP
}

\section{RESUMO}

Relata-se um caso de hérnia discal lombar associada a cisto extradural intra-raquidiano ("cisto discal"). Esse cisto, bem definido e homogêneo à tomografia, localizava-se na região ventrolateral extradural do espaço adjacente à hérnia discal lombar. A ressonância magnética com contraste demonstrou uma margem de realce, além de uma comunicação com a hérnia discal comprovada no ato cirúrgico.

PALAVRAS-CHAVE

Cisto discal. Disco intervertebral. Hérnia discal.

\begin{abstract}
Herniated lumbar disc associated to discal cyst. Considerations on diagnostic images and pathogenesis

We report a case of intraspinal extradural cyst communicating with an adjacent $L 5$ herniated disc which is nominated as "disc cyst". The cyst was well defined and homogeneous at computed tomography and was located in the ventrolateral extradural space adjacent to the herniated disc. It showed rim enhancement on contrast-enhanced MR images, besides a communication with the herniated disc as observed in the surgery.
\end{abstract}

\section{KEYWORDS}

Disc cyst. Herniated lumbar disc. Intervertebral disc.

\section{Introdução}

Lesões císticas intra-raquidianas extradurais são incomuns e incluem cistos sinoviais, cistos ganglionares, cistos perineurais de Tarlov, cistos extradurais da aracnóide, cistos dermóides e neuromas com formação cística.

Apresenta-se um caso de cisto intra-raquidiano extradural que se comunicava com hérnia discal lombar.

\section{Relato do caso}

LTS, 20 anos de idade, sexo masculino, comerciante, procedente de São Paulo, foi admitido no Ambulatório de Neurocirurgia do Hospital São Cristovão, em São Paulo, SP, com história de dor lombar na região posterior da perna esquerda que se iniciou três meses antes, ao acordar. A dor era em queimação e aperto, contínua, de moderada a forte intensidade, além de paroxismos de dor em choques, de freqüência variável; esses paroxismos prejudicavam as atividades diárias, obrigando-o a repouso ao leito. Atividades físicas, como deambulação e o ato de tossir ou espirrar pioravam as dores, que quando de forte intensidade, além da região posterior da perna esquerda, atingiam também o pé, sem melhora com analgésicos. Ao exame neurológico, notava-se apenas discreta hipoestesia na região posterior da perna esquerda e disestesia no território correspondente à inervação da raiz de S1. Havia uma diminuição do reflexo aquileu à esquerda, não havendo deficiência motora nem sinal de Lasègue.

$\mathrm{O}$ exame de raios $\mathrm{X}$ da coluna lombar realizado nas posições ântero-posterior e perfil demonstrou-se dentro da normalidade e a tomografia computadorizada (TC) realizada com cortes axiais da região espaço discal L5-S1 demonstrou uma imagem de aspecto semelhante à hérnia discal, situada anterior e lateralmente à

*Serviço de Neurocirurgia do Hospital São Cristovão, São Paulo, SP.

**Serviço de Patologia Landman, São Paulo, SP.

***Neurorradiologista do Laboratório Fleury, São Paulo, SP. 
esquerda do saco dural, com bordos bem delimitados (Figura 1). ATC com janela óssea não revelou sinais de erosão óssea na região acometida (Figura 2).

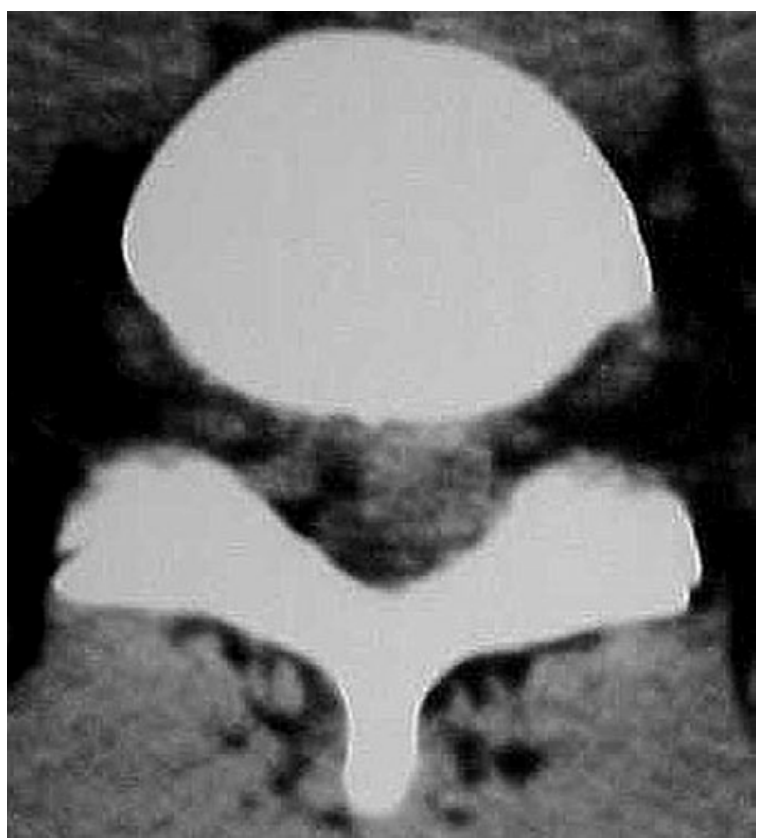

Figura 1 - TC do espaço discal L5 demonstrou uma imagem de aspecto semelhante à hérnia discal, situada anterior e lateralmente à esquerda do saco dural.

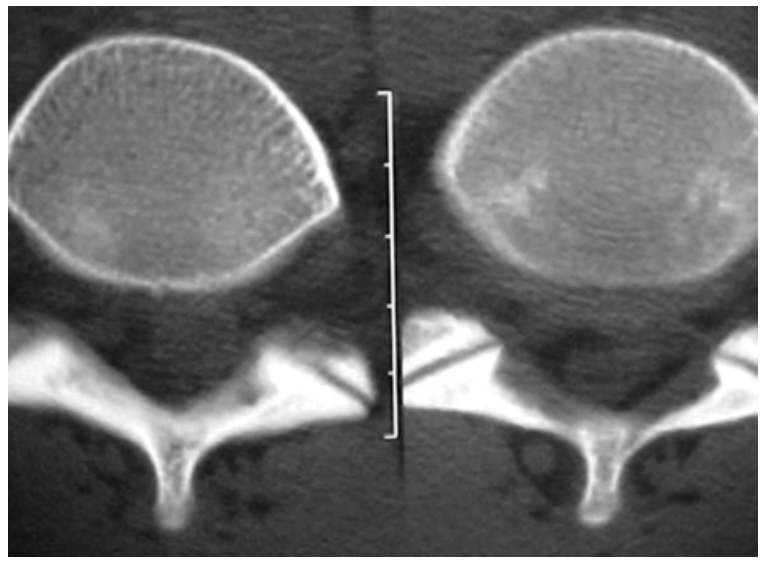

Figura 2 - TC com janela para osso não revelou sinais de erosão óssea na região acometida.

A ressonância magnética (RM) de coluna lombar, ponderada em T1 e corte sagital, mostrou uma massa hipointensa causando compressão do saco dural entre L5-S1 (Figura 3) que se revelou hiperintensa em T2 (Figura 4); houve captação de contraste nos bordos da massa, que apresentava forma arredondada e que comprimia o saco dural anterior e lateralmente à esquerda e, no corte axial, sugeria haver continuidade com o disco de L5 (Figura 5).

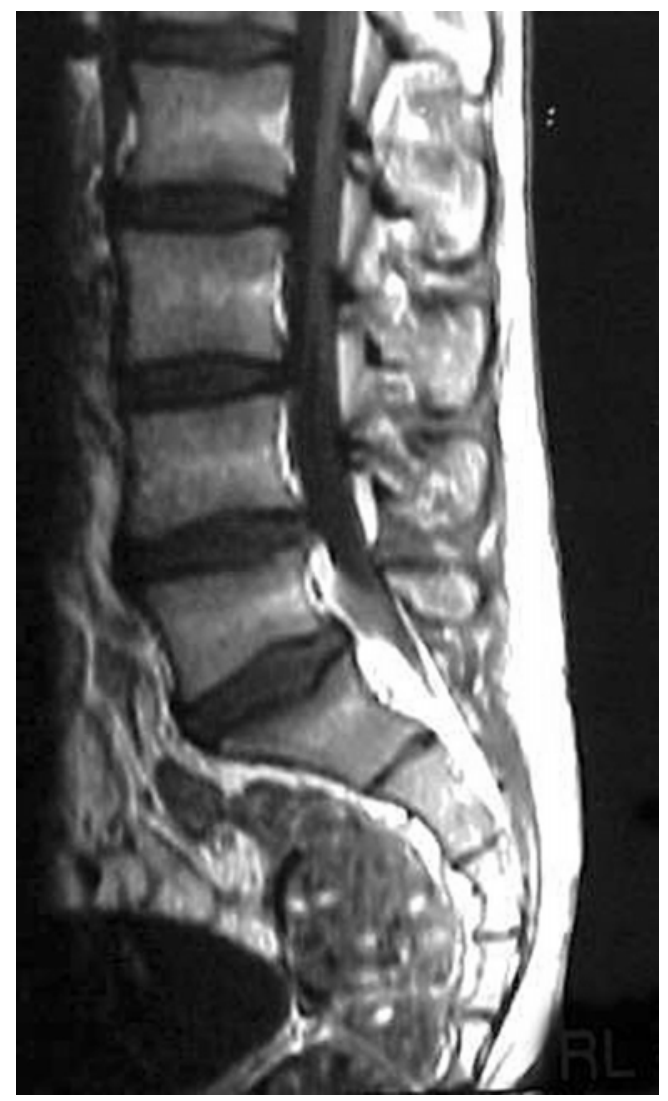

Figura 3 - RM ponderada em T1 mostrou uma massa hipointensa causando compressão do saco dural no espaço discal L5-S1.

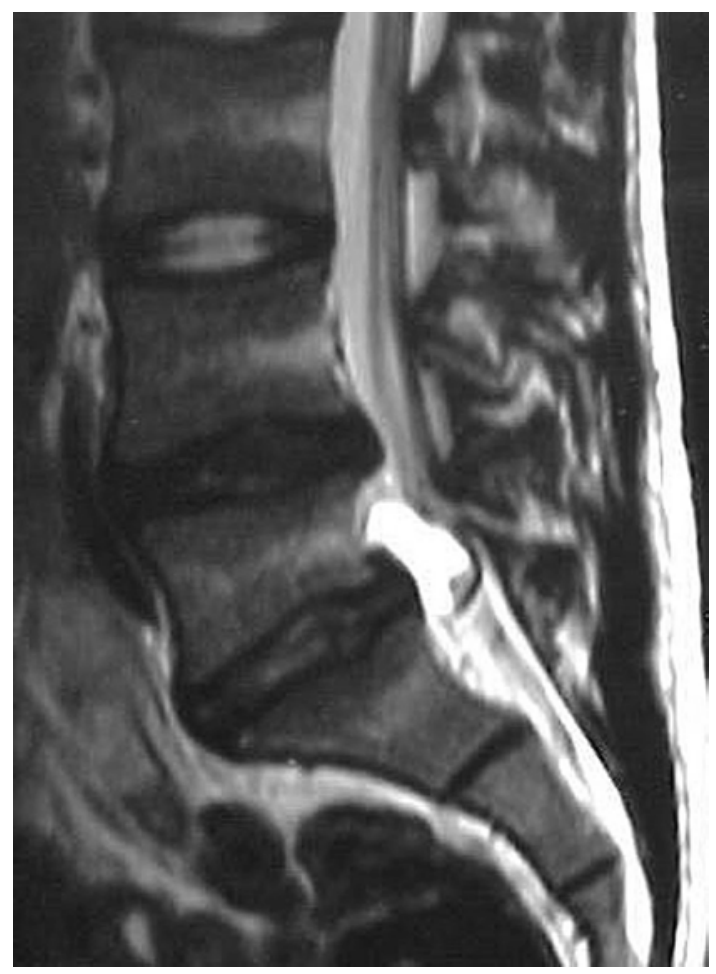

Figura 4 - A mesma lesão revelou-se hiperintensa em T2. 


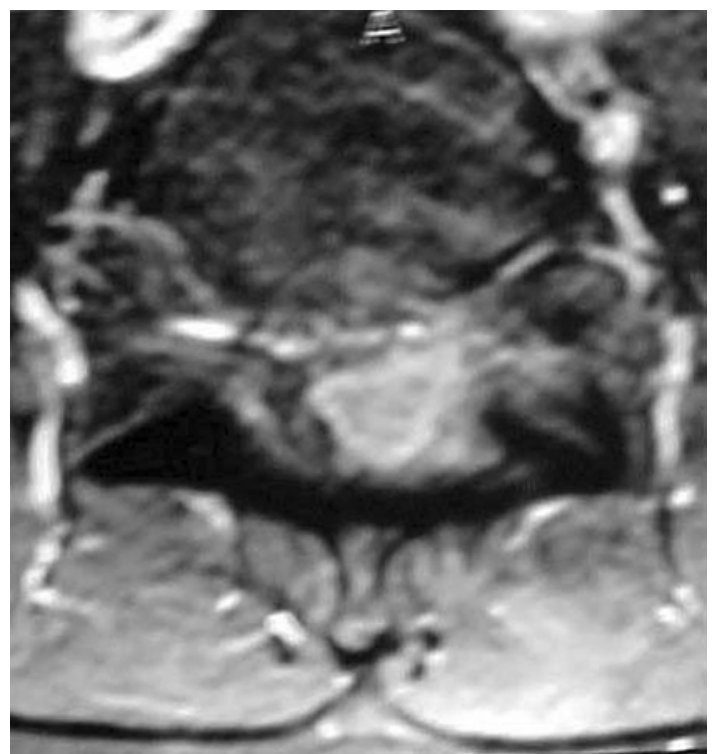

Figura 5 - RM em corte axial, mostrando captação de contraste nos bordos da massa que apresentava forma arredondada e que comprimia o saco dural anterior $e$ lateralmente à esquerda, além de sugerir continuidade com o disco de L5.

O paciente foi submetido à laminectomia de L5. Um cisto foi observado adjacente à região posterior ao corpo vertebral de L5, comprimindo a raiz do nervo de L5 à esquerda e dorsalmente (Figura 6). O cisto comunicava-se com o disco envolvido através de um pequeno orifício e continha um líquido de aspecto "petroláceo" semelhante ao encontrado nas drenagens de hematoma subdural crônico. O cisto e o disco de L5 foram retirados deixando livre de compressão as raízes de L5 e S1. O exame histológico da parede do cisto revelou tecido conectivo fibrótico, sem a presença de células sinoviais; observou-se a presença de proliferação vascular na parede do cisto de tecido conectivo fibrótico (Figuras 7, 8 e 9).

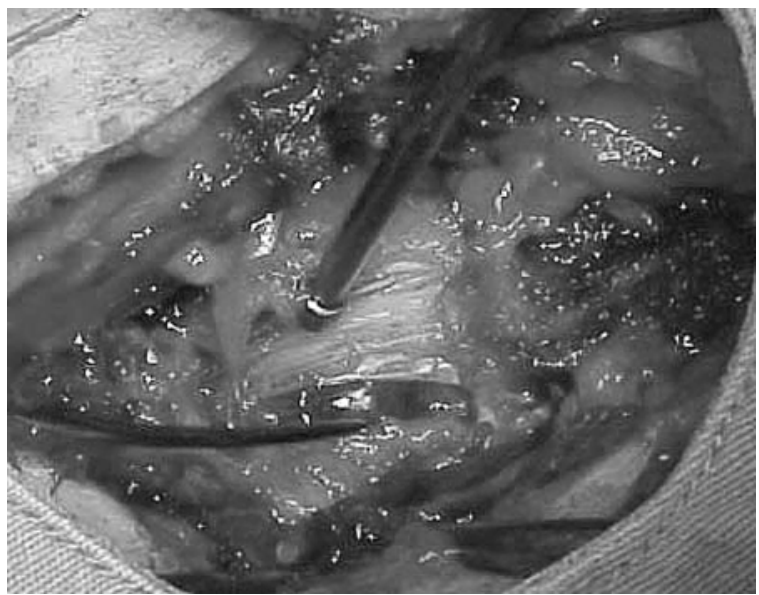

Figura 6 - Após a laminectomia de L5, foi observado um cisto adjacente à região posterior ao corpo vertebral de $\mathrm{L5}$, comprimindo a raiz L5 à esquerda e dorsalmente.

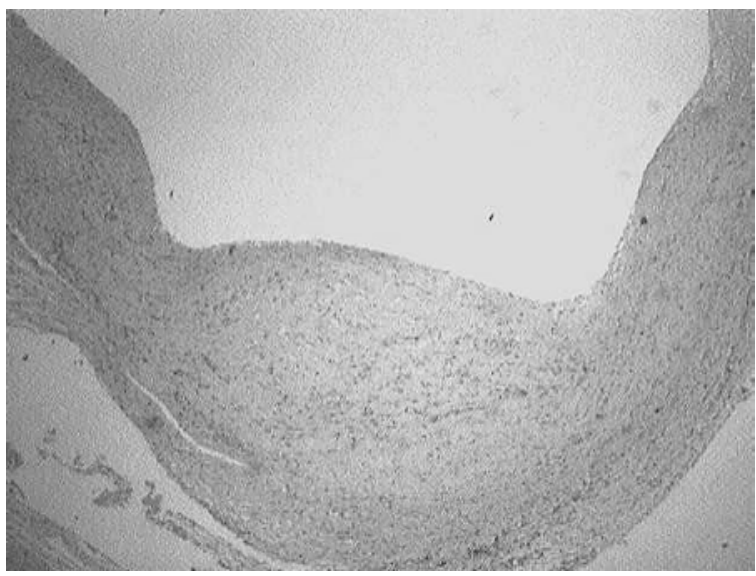

Figura 7 - Aspecto histopatológico do cisto, com a parede destituída de revestimento epitelial (H\&E 25X).

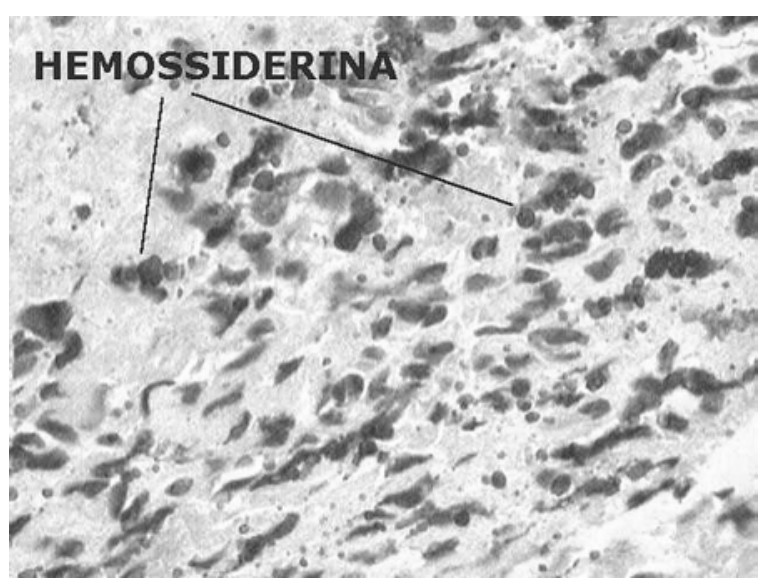

Figura 8 - Area degenerativa com focos de deposição de hemossiderina, indicando hemorragia pregressa (H\&E 200X).

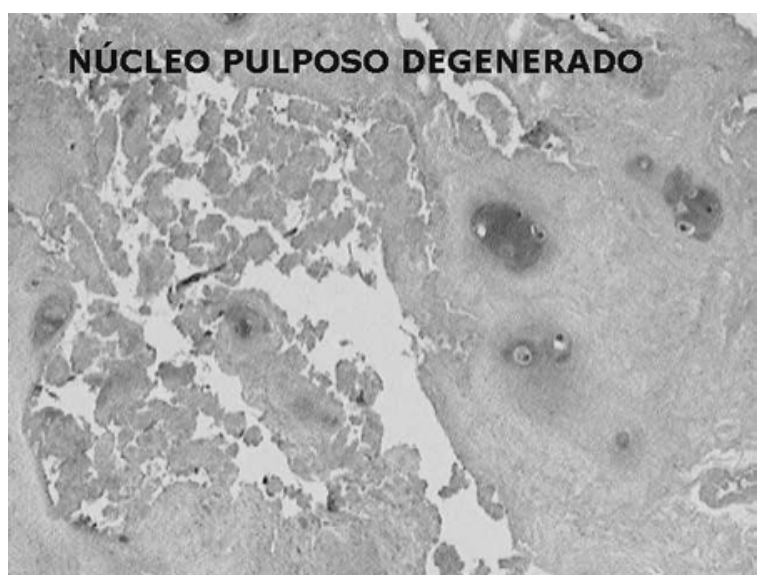

Figura 9 - Fotomicrografia do núcleo pulposo exibindo cartilagem com áreas de degeneração e fragmentação (H\&E 200X). 
O paciente evoluiu com melhora da dor, recebendo alta hospitalar no terceiro dia do pós-operatório, sem uso de medicação analgésica.

\section{Discussão}

A comunicação do cisto com a hérnia discal é denominada "cisto discal"'. Em 1997, houve a publicação de um caso semelhante, a esse relatado, no Japão ${ }^{10}$. Um cisto discal bem definido e homogêneo, apresentando-se no espaço extradural ventrolateral adjacente à hérnia discal lombar deslocando o saco dural dorsomedialmente. Na RM, a massa cística era homogênea e isointensa ao líquor e havia captação de contraste demarcando o contorno da lesão cística.

O mecanismo de formação do cisto é desconhecido. Duas hipóteses têm sido sugeridas. A primeira implica formação de um hematoma epidural, já que o conteúdo cístico continha fluido hemorrágico em quatro dos sete casos apresentados por Toyama e cols. ${ }^{10}$. Essa hipótese sugere que o hematoma epidural é produzido pela ruptura de veias epidurais pelo mecanismo de irritação inflamatória da hérnia discal. De acordo com Toyama e cols. ${ }^{10}$, o hematoma eventualmente torna-se um cisto discal. Hematomas epidurais intra-raquidianos têm sido publicados ${ }^{4}$ e os achados de imagem são similares aos achados de outros casos relatados com relação à localização e à definição do cisto. Entretanto, Kono e cols. ${ }^{6}$ referem que não há captação de contraste na RM ou na discografia e que a comunicação do cisto com o disco não é documentada; além disso, ressalta o fato de o cisto poder conter material seroso e de que a parede do cisto, nos exames de microscopia, não revela hemossiderina, não dando suporte à hipótese do hematoma epidural.

Kono e cols. ${ }^{6}$ sugerem a segunda hipótese, pela qual a formação do cisto discal se deve a uma degeneração focal do disco e que o cisto conduz a um amolecimento do colágeno do disco, levando à formação do líquido, similar ao mecanismo de formação do cisto meniscal em pacientes com degeneração do menisco no joelho ${ }^{2}$. O disco intervertebral degenerado leva à formação do conteúdo do cisto. A formação da pseudomembrana e o encapsulamento do conteúdo fluido é causado de forma semelhante ao cisto meniscal (Figura 10). O achado histológico da parede do cisto, incluindo estruturas fibróticas sem a presença de células sinoviais, são compatíveis com uma pseudomembrana. O fluido do cisto é seroso, podendo conter proteínas degradadas que assumem aspecto semelhante ao material discal degenerado.

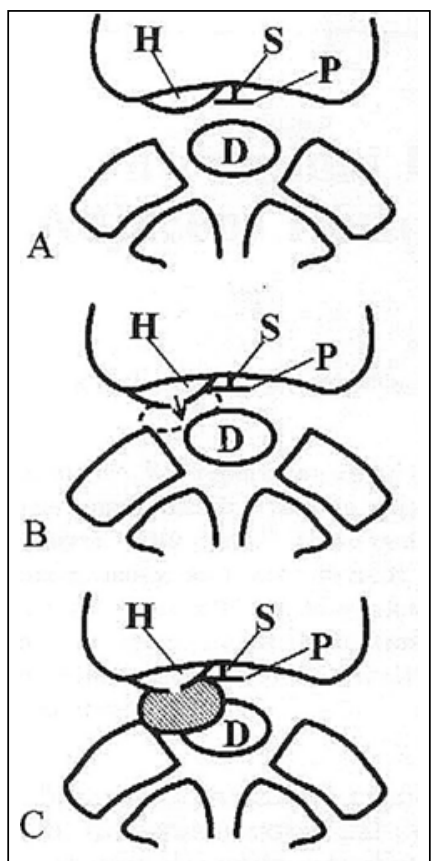

Figura 10 - Ilustração do mecanismo de formação do cisto discal: A: Desenvolvimento da hérnia de disco;

B: Degeneração do material discal gerando a formação do fluido (seta). A linha tracejada indica a pseudomembrana;

C: Relação da hérnia de disco com o cisto discal. A área pontilhada indica o cisto discal. $A$ interrupção na parede do cisto indica a comunicação do cisto com a hérnia discal. Legendas: $D=$ saco dural, $H=$ hérnia de disco,

$P=$ ligamento longitudinal posterior, $S=$ septo medial.

O diagnóstico diferencial clínico e radiológico das lesões de massas císticas extradurais intra-raquidianas inclui: cisto sinovial, cisto ganglionar, cisto perineural de Tarlov ${ }^{9}$, cisto aracnóideo extradural ${ }^{3}$, cisto dermóide e neuroma com alterações císticas. $\mathrm{O}$ cisto sinovial desenvolve-se da junção facetária ${ }^{7}$, é usualmente de localização póstero-lateral em relação ao saco dural, contendo fluido mucinoso e há presença de células sinoviais na parede do cisto ${ }^{5}$. Os cistos ganglionares, de origem discutida, correpondem usualmente a gânglios que aumentam a junção facetária e situam-se dorsolateralmente ao saco dural; histologicamente, a parede do gânglio é feita de tecido conectivo sem a presença de células sinoviais, mas, usualmente, a anatomia patológica diferencia o cisto ganglionar do cisto discal e, este, freqüentemente provoca erosão óssea do corpo vertebral, além de deslocar o saco neural. A parede do cisto ganglionar é composta por estroma frouxo, com células estreladas, sem revestimento epitelial ${ }^{8}$. O diagnóstico diferencial entre essas duas afecções citadas é difícil; a discografia pode ajudar no diagnóstico diferencial, pois mostra a comunicação do cisto com o disco envolvido. Os cistos peridurais de Tarlov são cistos derivados das meninges; são freqüentemente múltiplos e podem ocorrer ao longo da 
raiz do nervo acometido. As fibras do nervo situam-se juntamente com a parede do cisto. Eles normalmente não captam contraste na $\mathrm{RM}^{1}$, fazendo-o somente quando há processo inflamatório associado. Os achados microscópicos do cisto aracnóideo extradural são semelhantes ao dos cistos discais; entretanto, ocorre mais freqüentemente em jovens, na região torácica e posteriormente ao saco dural e não captam contraste nas imagens da $\mathrm{RM}^{3}$. Os neuromas císticos têm, freqüentemente, imagens semelhantes ao do cisto discal, mas, na maioria das vezes, apresentam aspecto com áreas nodulares ou com captação heterogênea de contraste.

Os cistos discais, embora raros, devem ser tratados rapidamente. A sua localização ventrolateral é o fator que mais o diferencia nas imagens de TC e de RM.

\section{Referências}

1. ARAKI Y, TSUKAGUSHI I, ISHIDA T: MRI of symptomatic sacral perineural cyst. Rad Med 10:2502, 1992.

2. CHEN WC, WU JJ, CHANG CY, CHEN BF, YANG DJ: Computed tomography of a meniscal cyst. Orthopedics 10:1569-72, 1987.

3. CLOWARD RB, BUCY PC: Spinal extradural cyst and kyphosis dorsalis juvenilis. Am J Roentgenol 38:681706, 1937
4. GUNDRY CR, HEITHOFF KB: Epidural hematoma of the lumbar spine. 18 surgically confirmed cases. Radiology 187:427-31, 1993.

5. HEMMATIM, THOMAS C, PATEL DV: Symptomatic intraspinal ganglion cyst of the nerve root sheath. AJNR Am J Neuroradiol 10:S100, 1989.

6. KONO K, NAKAMURA $\mathrm{H}$, INOUE $\mathrm{Y}$, OKAMURA T, SHAKUDO M, YAMADA R: Intraspinal extradural cysts communicating with adjacent herniated disk : characteristics and possible pathogenesis. AJNR 20: 1373-7, 1999.

7. LIU SS, WILLIAMS KD, DRAYER BP, SPETZLER RF, SONNTAG VKH: Synovial cyst of the lumbasacra spine. Diagnosis by MR imaging. AJNR 10:1239-42, 1989.

8. OGAWA Y, KUMANO K, HIRABAYASHI S, AOTA Y: A ganglion cyst in the lumbar spine canal. A case report. Spine 17:1429-31, 1992.

9. TARLOV IM: Perineural cyst of the spine nerve roots. Arch Neurol Psychiatry 51:125-46, 1938.

10. TOYAMA Y, KAMATA N, MATSUMOTO M: Pathogenesis and diagnostic title of intraspinal cyst communication with intervertebral disk in the lumbar spine. Rinsho Seikei Geka 32:393-400, 1997.

Original recebido em outubro de 2001

Aceito para publicação em junho de 2002

\section{Endereço para correspondência:}

Marcelo Ferraz de Campos

Rua Pedro de Toledo, 541, ap. 44

CEP 04039-031 - São Paulo, SP

E-mail:ferrazcampos@uol.com.br 